\title{
Lightons: Phonons with light
}

\author{
Nuno A. Silva ${ }^{a, b}$, M. I. Carvalho ${ }^{c}$, A. Guerreiro ${ }^{a, b}$ \\ a INESC TEC - UOSE, R.Campo Alegre, 4169-007, Porto, Portugal. \\ b Faculdade de Ciências da Universidade do Porto, R.Campo Alegre, 4169-007, Porto, \\ Portugal. \\ c Faculdade de Engenharia da Universidade do Porto, R. Dr. Roberto Frias, 4200-465 Porto, \\ Portugal
}

\begin{abstract}
Spatial solitons are robust localized nonlinear waves that are able to propagate without significant changes to their structure. Most of the proposal of the application of solitons uses them to transmit and process information in optical fibers and optical circuits. In the later the solitons can be guided through different paths by presetting some soliton characteristics (such as the phase), and even using some solitons to control the path of other pulses. In this paper, we use these properties of optical spatial solitons in a cubic nonlinear media to have lightons: phonon-like oscillations of a chain of solitonic light pulses. Conceptually, this work aims to explore the dual nature of solitons as a particle-like wave, by considering the displacement wave of solitons in a 1-dimensional chain.
\end{abstract}

Keywords: Solitons, Nonlinear Optics

\section{INTRODUCTION}

The nature of solitons exists between wave and particle. At a fundamental point of view, soliton appear as stable solution of nonlinear wave equations, such as the Nonlinear Schrodinger equation (NLSE), balancing wave dispersion with nonlinear wave interaction, and resulting in a coherent wave package that can behave both like a particle and a wave. For example, depending on the relative global phase of the soliton and the type of nonlinear media, two optical solitons can scatter each other like two rigid bodies, ${ }^{1-4}$ go right through each other like a wave,$^{5}$ or coalescence in a wider soliton like two droplets of a liquid. ${ }^{6}$ The versatility of behavior found in solitons has captured the interest in mathematics and physics and can be used to describe many phenomena in different fields of science. In this paper we consider solitons of the electromagnetic field in nonlinear optical media. However the underlying model of mathematical physics has a wider validity beyond nonlinear optics, and may describe problems in fluid dynamics, plasma physics, protein chemistry and more. ${ }^{1,3}$

Just as waves can exhibit particle like behavior, particles can also support waves, such as mechanical waves. For example, a chain of interacting particles can display collective oscillatory motions which depend on the nature of the interaction forces. Elastic linear interaction allow small displacements of the particles from their equilibrium positions to form mechanical waves, know as phonons, that propagate throughout the chain. As waves, phonons can not only propagate but also superpose and interfere.

From this duality, where nonlinear wave packages display particle behavior and particles can support waves, arises a question: can solitons aligned in a chain support mechanical waves similar to phonons? Or, in a simpler way: can we have waves made of particles which are made of waves? The answer to this is not easy. It is necessary to combine several ingredients just the right way, which include the type of nonlinearity and type of solitons, their relative phase, their parameters, their interaction, etc.. Then, when all the conditions are met, we may have a type of mechanical wave that we call lightons.

In this paper we focus our attention in the collective oscillations for an 1-dimensional chain of $\mathrm{N}$ optical solitons, supported by a medium with a cubic non-linearity and described by the NLSE. In section 2, following a quasiparticle approach to describe the soliton interaction, is derived a complete set of equations for the evolution of the parameters of each soliton, including their positions and global phase. We show that in the adiabatic 
regime these equations reduce to the Toda Lattice equation (TLE) ${ }^{7}$ that in small amplitude limit can support phonon-like solutions. ${ }^{8}$ In section 3 we discuss the numerical methods used to simulate the system and how the use of the graphics processor unit (GPU) can boost this type of studies. In section 4, we analyze the stability and dispersion of displacement waves along the soliton chain using numerical methods and compare them with the results for the TLE. Finally, we present the possibility of transposing this collective behavior to spatial solitons in a 2-dimensional system, supported by cubic-quintic nonlinearities.

\section{PHYSICAL MODEL}

The evolution of optical solitons in a media with cubic non-linearity is governed by the (1+1)-dimensional NLSE

$$
i \frac{\partial v}{\partial z}+\frac{1}{2} \frac{\partial^{2} v}{\partial x^{2}}+|v|^{2} v=0
$$

where $z$ is the propagation distance and $x$ is coordinate along the transverse direction. The single soliton solution for this equation is

$$
v_{j}(z, x)=2 \nu_{j} \operatorname{sech}\left\{2 \nu_{j}\left(x-\overline{x_{j}}\right)\right\} \exp \left\{i 2 \mu_{j}\left(x-\bar{x}_{j}\right)+i \delta_{j}\right\}
$$

where the parameters $\nu_{j}, \bar{x}_{j}, \mu_{j}$, and $\delta_{j}$ refer to the amplitude, the position, the frequency and the phase of the soliton.

We consider the limit of large distance and small overlap between consecutive solitons, where the N-soliton solution for the equation (1) can be given as a sum of $\mathrm{N}$ single soliton solutions

$$
v(z, x)=\sum_{j=1}^{N} v_{j}(z, x)
$$

When replacing (3) into equation (1) it becomes clear that the nonlinear term is responsible for the interaction between solitons. To solve the resulting equation, we use an ansatz and separate equation (1) into the following $\mathrm{N}$ coupled equations, one for each single soliton:

$$
i \frac{\partial v_{j}}{\partial z}+\frac{1}{2} \frac{\partial^{2} v_{j}}{\partial x^{2}}+\left|v_{j}\right|^{2} v_{j}=i R_{j}[v]
$$

It is simple to see that the sum of the solutions of each of these equations is a solution of equation (1). Each of these equations is just a NLSE with a small perturbation $R_{j}$ associated to solitonic interaction. Considering that only first neighbors can interact and expanding in the first order of the overlap $u_{j} u_{j+1}^{*}$ we get

$$
R_{j}[v]=2\left|v_{j}\right|^{2}\left(v_{j+1}+v_{j-1}\right)+v_{j}^{2}\left(v_{j+1}^{*}+v_{j-1}^{*}\right)
$$

The initial problem is now reduced to a form which is suitable to be analyzed using a quasiparticle approach which results in the following evolution equations for the parameters of each j-th soliton ${ }^{7}$

$$
\begin{gathered}
\frac{d \mu_{j}}{d z}=16 \nu^{3}\left[\cos \left(\phi_{j+1, j}\right) \exp \left(-\Delta_{j, j+1}\right)-\cos \left(\phi_{j-1, j}\right) \exp \left(-\Delta_{j-1, j}\right)\right] \\
\frac{d \overline{x_{j}}}{d z}=2 \mu_{j}-4 \nu\left[\sin \left(\phi_{j+1, j}\right) \exp \left(-\Delta_{j, j+1}\right)-\sin \left(\phi_{j-1, j}\right) \exp \left(-\Delta_{j-1, j}\right)\right] \\
\frac{d \delta_{j}}{d z}=2\left(\nu^{2}+\mu_{j}^{2}\right)+2 \mu\left(\frac{d \overline{x_{j}}}{d z}-2 \mu_{j}\right)+24 \nu^{2}\left[\cos \left(\phi_{j+1, j}\right) \exp \left(-\Delta_{j, j+1}\right)+\cos \left(\phi_{j-1, j}\right) \exp \left(-\Delta_{j-1, j}\right)\right]
\end{gathered}
$$

Here $\phi_{j, l}=2 \mu\left(\bar{x}_{l}-\bar{x}_{j}\right)+\psi_{j, l}$ is the complex phase between solitons, with $\psi_{j, l}=\delta_{j}-\delta_{l}$, the quantity $\Delta_{j, l}=$ $2 \nu\left|\overline{x_{l}}-\overline{x_{j}}\right|$ is the spacing between two solitons and $\nu$ and $\mu$ are the mean values for the amplitude and frequency, respectively. The derivation of these equations takes into account the assumptions that the overlap is small, i.e. $\nu\left|\bar{x}_{l}-\bar{x}_{j}\right| \gg 1$, and that the solitons have similar frequencies and amplitudes, i.e. $\left|\mu_{j}-\mu_{l}\right| \ll \mu,\left|\nu_{j}-\nu_{l}\right| \ll \nu$. Also it is assumed that the amplitude $\nu_{j}$ of each soliton is approximately constant, a fact which occur in the adiabatic limit and is supported by small amplitude variation observed in numerical simulations. 
We also adopt a conjecture commonly made, ${ }^{7}$ where it is assumed that if the system is initialized with consecutive solitons having a phase difference of 0 or $\pi$, this difference remains constant during the motion of the solitons. This assumption leads us to the TLE

$$
\frac{\partial^{2} u_{j}}{\partial z^{2}}=32 \cos (\Psi) \nu^{3} \exp (-2 \nu \Delta)\left\{\exp \left(-2 \nu\left(u_{j+1}-u_{j}\right)\right)-\exp \left(-2 \nu\left(u_{j}-u_{j-1}\right)\right)\right\}
$$

where $u_{j}=\bar{x}_{j}-x_{j}^{0}$ is the displacement of the $j-t h$ soliton from its position of equilibrium $x_{j}^{0}$. The phase difference between consecutive solitons is represented by $\Psi$ and the equilibrium lattice spacing is $\Delta=x_{j+1}^{0}-x_{j}^{0}=L / N$ with $[0, L]$ the limits of the simulation box. We impose the later condition considering that our problem has periodic boundary conditions and that each soliton is at a stable point of the lattice.

For small displacements we can expand the TLE as

$$
\frac{\partial^{2} u_{j}}{\partial z^{2}} \simeq C\left(u_{j+1}-2 u_{j}+u_{j-1}\right)+\mathcal{O}\left(u^{2}\right)
$$

with the constant $C$ given by $C=-64 \cos (\Psi) \nu^{4} \exp (-2 \nu \Delta)$. This equation resembles the equation of motion for collective oscillations - phonons - which have the traveling waves solutions

$$
u_{j}=\sum_{k} A_{n} e^{-i\left(k x_{j}^{0}-\omega_{k} t\right)}
$$

obeying the dispersion relation

$$
\omega_{k}=\sqrt{\frac{2 C}{M}(1-\cos k \Delta)}
$$

that implies that $C>0$ and then $\Psi=\pi$.

It is important to discuss the validity of the assumption that the relative phase between consecutive solitons $\Psi$ remains constant throughout the evolution of the system. Equation 8 implies that the phase of a moving soliton must change. Since the motion of two consecutive solitons is different then, their phase difference cannot remain constant. Therefore equation 9 and 8 are inconsistent, ${ }^{7}$ fact also stated by Novoa et. al ${ }^{9}$ and leads them to consider an analog ideal binary system that eliminates the phase difference dependence fixing the interaction between consecutive solitons.Due to this questions and also to the non-linearity of the TLE it seems odd to expect a phonon-like behavior with traveling waves as normal modes. However, even knowing that the normal modes purposed above cannot describe perfectly the dynamics of the system, we expect that for small displacements and short evolutions the solitons can support collective oscillations. With the evolution of the system, there is an accumulation of phase difference between solitons, which changes the nature of their interaction, resulting in a distortion of the phonon-like modes and ultimately destroying them. In fact, the phase accumulation can be seen as a phase transition where the solitons stop interacting as particles in a chain and resulting into a new type of collective behavior.

\section{NUMERICAL METHODS}

The system is simulated using the well-known split step Fourier method ${ }^{10}$ (SSFM) to solve the NLSE (1) given the initial conditions for the soliton chain. The implemented method takes advantages from the high performance of the graphics processor unit (GPU) and the CUDA ${ }^{11}$ libraries to speed the code up to 6 times faster than a CPU based code for the same problem. This speedup is significant as the number of steps needed to properly analyze the system is big.

In the next section we will present numerical results for a chain of $N=40$ solitons with the initial condition

$$
v=\sum_{j=1}^{N} 2 \nu \operatorname{sech}\left\{2 \nu\left(x-x_{j}^{0}-u_{j}\right)\right\} e^{i j \pi}
$$


where $x_{j}^{0}=\left(j+\frac{1}{2}\right) \Delta$. The $u_{j}$ are chosen as $u_{j}=\sin (k j \Delta)$ with $k=2 \pi n / N$ the wave number for this wave. Initializing the soliton frequencies as $\mu_{j}=0$, we expect solutions of the type of standing waves, which will led us to the dispersion relation $\omega(k)$ that could be compared with the theoretical dispersion defined in equation (12).

The GPU used is a standard GeForce 640M with 384 CUDA cores. In all 1-dimensional simulations reported in the next section will be used the values $\Delta=20$ and $\nu=0.35$ the for soliton parameters. The spatial discretization used was $d x=800 / 2^{11}$ and the integration step was $d h=0.02$. The typical simulation time is about 11 minutes.

\section{RESULTS AND DISCUSSION}

Figure 1 shows the results of the simulations for standing waves in the solitonic chain with wave number equal to $k=2 \pi n / N$ with $n=3$ and $n=15$. In the insets of figure 1a) and 1d) we can identify in more detail the oscillatory and wave-like motion of the solitons. We have chosen small oscillation amplitudes $A=0.5$, compared to the distance between solitons, to satisfy the validity conditions of the model described in section 2 .
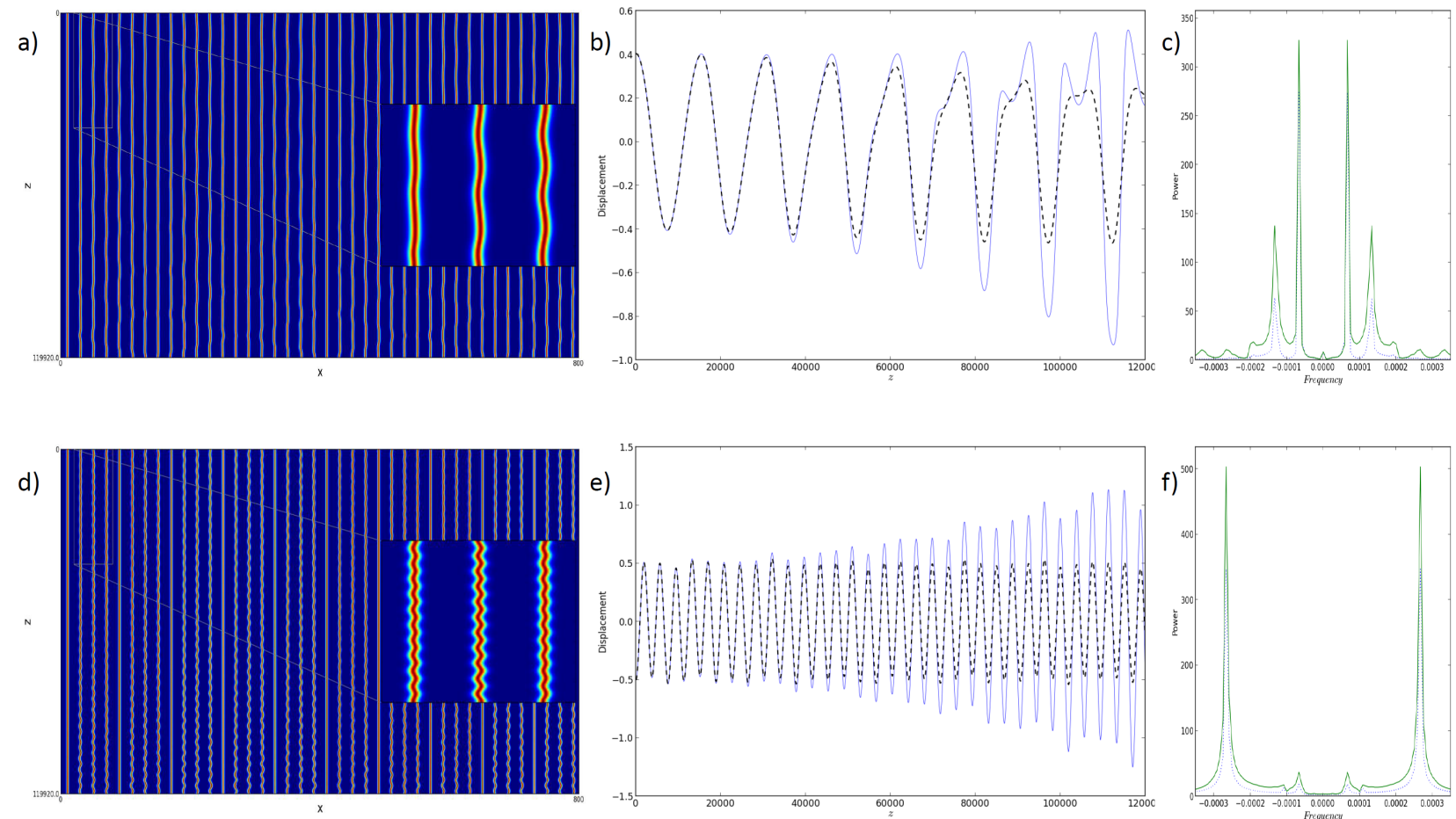

Figure 1. Evolution of the soliton chain for $n=3(\mathrm{a}), \mathrm{b}), \mathrm{c}))$ and $n=15(\mathrm{~d}), \mathrm{e}), \mathrm{f}))$ ). Figures a) and d) show the intensity profile evolution where we can identify the phonon-like oscillations. Figures b) and e) compare in detail the displacement of the second soliton from the left, obtained from the simulations (solid line) with the prediction of TLE (dashed line). Finally figures c) and f) display the Fourier transform of the displacements wave (numerical results with solid line, TLE with dashed line), where we can identify the generation of new frequencies for lower $n$.

In figure 1b) and 1e) we can compare the motion of a soliton observed in the simulations with that predicted by the Toda model. Clearly, the match is closer for larger $n$ where the phonon-like motion is more stable. In both cases the amplitude of the oscillation increases during the evolution, implying that the system is not really undergoing a periodic motion. However, the characteristic time of the oscillation, or pseudo-period, defined as the period of return of the soliton to the position of equilibrium $u=0$, remains stable throughout the simulation. For $n=3$, the nonlinear character of equation (9) is stronger and leads to the generation of higher harmonics in the spectrum of the motion of the soliton, as seen in figure 1c).

Figure 2 allows to verify the limitation of the TLE model by comparing the absolute difference between the displacement predicted and the calculated in the simulations, with the deviation of the phase difference between 
consecutive solitons from its initial value $\pi$. Clearly there is a good correlation which indicate the connection between the accumulation of error in phase difference and degradation of the phonon-like oscillations.

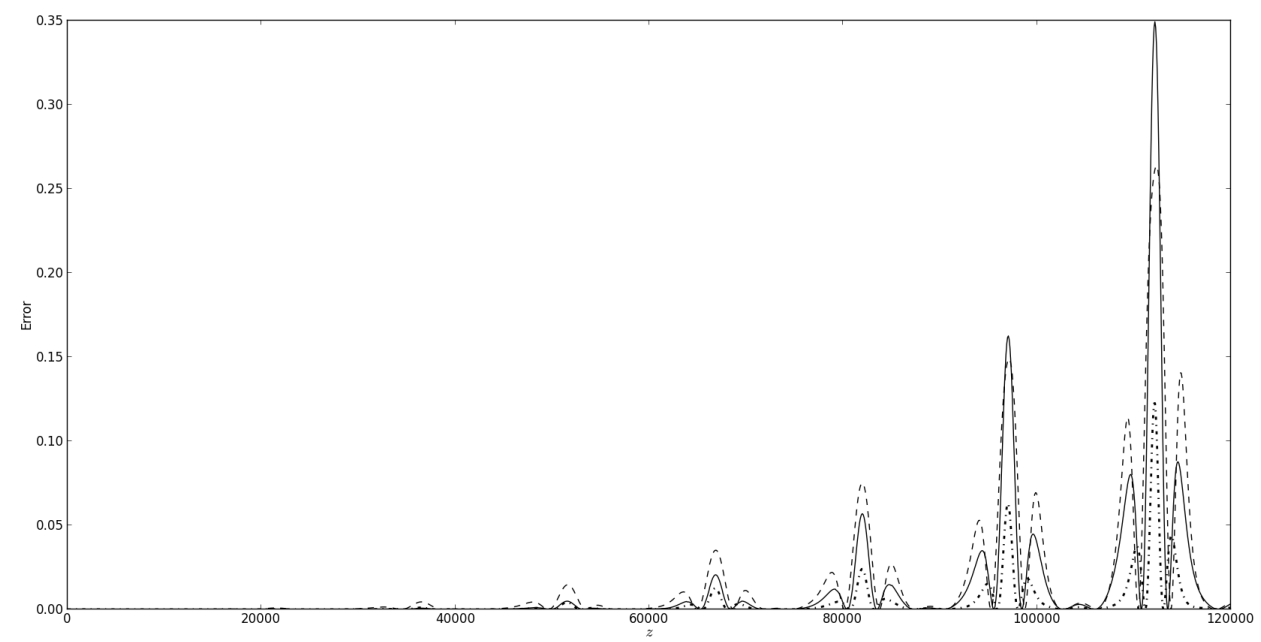

Figure 2. Error analysis of the displacement wave for a soliton between the numerical simulation and TLE model. Solid line is the absolute value of displacement error, $\left|u_{2}-u_{2}^{T L E}\right|$, dashed and dash-point line is the absolute value of error for phase difference between consecutive solitons and the prediction $\left|\psi_{2,3}-\pi\right|,\left|\psi_{1,2}-\pi\right|$ respectively.

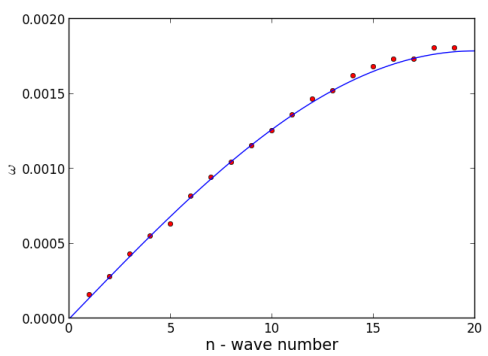

Figure 3. Dispersion relation computed for a chain of $N=40$ solitons (circular markers) and comparison with the TLE model (solid line).

Simulating standing waves for parameter $n \in] 0,20[$ we can obtain the right-side branch of the dispersion relation, shown in figure 3. As we can see, the data obtained from the pseudo-period values for each wave number leads to the expected theoretical dispersion, what confirms again the possibility of having standing waves propagating in chains of solitons, revealing a new hierarchy in the wave-particle duality. Similar results can be obtained for chains with as many solitons as wanted.

Finally we show that these effects are not limited to soliton chains in 1-dimension but can be generalized to higher spatial dimensions. In figure 4 we show a chain in a 2-dimensional space. Here the spatial solitons are Gaussian shapes supported by a cubic-quintic media and the early results show that it is possible to propagate energy and momentum with wave-like phenomena in a chain of solitons.

\section{CONCLUSIONS}

We have explored the dynamics of an 1-dimensional chain of optical solitons in a cubic nonlinear media described by the NLSE. Using perturbative methods we show that the interaction between consecutive solitons could support wave-like oscillations of the positions of the optical solitons in the chain. We call these waves lightons: 
phonons of light. Numerical simulations show it is possible to create standing wave oscillations in the considered system that follows a predicted relation of dispersion. For small propagation distances and displacement amplitudes, the system revealed a controllable sinusoidal motion for the soliton position which is obtained only with light-light interaction. Then, our results suggests the possibility of steering spatial solitons with other solitons, a property that might be employed in practice to control the guidance of light in fully optical devices.

a)

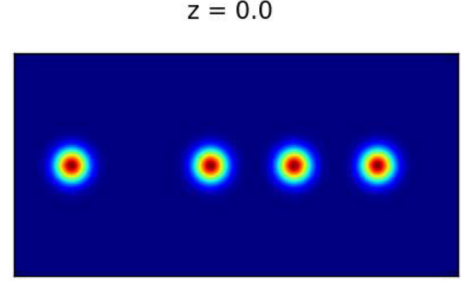

$$
z=80.0
$$

b)

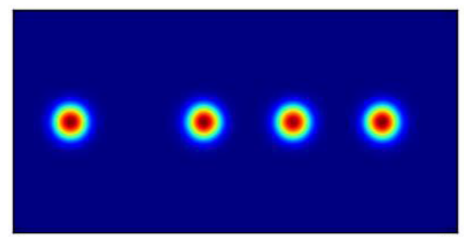

$z=160.0$

c)

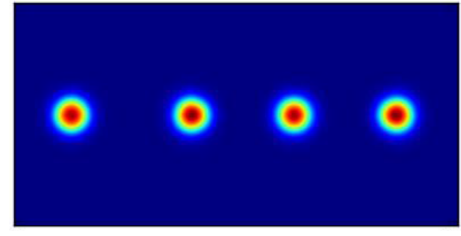

$z=240.0$

d)

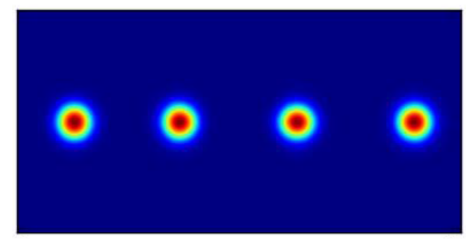

$z=320.0$

e)

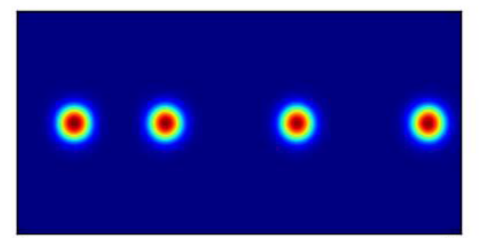

f)

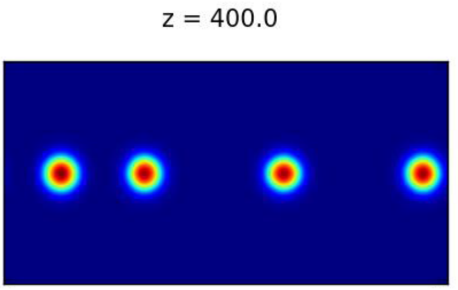

$z=480.0$

g)

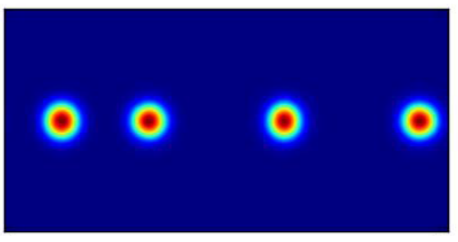

$z=560.0$

h)

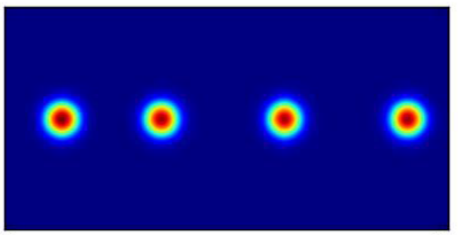

$z=640.0$

i)

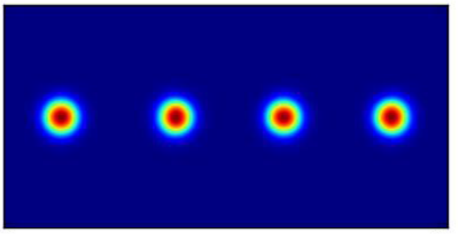

$z=720.0$

j)

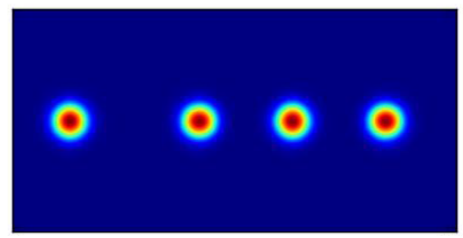

Figure 4. Evolution of an 1-dimensional chain of 2-dimensional spatial solitons with $\Delta=20$. 


\section{REFERENCES}

1. Kivshar, Y.S. and Malomed, B. A., "Dynamics of solitons in nearly integrable systems," Rev. Mod. Phys. 61, 763-915 (Oct 1989).

2. Yang, J. and Tan, Y., "Fractal structure in the collision of vector solitons," Phys. Rev. Lett. 85, 3624-3627 (Oct 2000).

3. Kivshar, Y. S., Agrawal, G. P., and Agrawal, G. P., "Chapter 1 and 2," in [Optical Solitons], 1 - 30, Academic Press, Burlington (2003).

4. Chamorro-Posada, P. and McDonald, G. S., "Spatial kerr soliton collisions at arbitrary angles," Phys. Rev. $E$ 74, 036609 (Sep 2006).

5. Aossey, D. W., Skinner, S. R., Cooney, J. L., Williams, J. E., Gavin, M. T., Andersen, D. R., and Lonngren, K. E., "Properties of soliton-soliton collisions," Phys. Rev. A 45, 2606-2610 (Feb 1992).

6. Michinel, H., Paz-Alonso, M. J., and Perez-Garcia, V. M., "Turning light into a liquid via atomic coherence," Phys. Rev. Lett. 96, 023903 (Jan 2006).

7. Uzunov, I., Gerdjikov, V., Golles, M., and Lederer, F., "On the description of n-soliton interaction in optical fibers," Optics Communications 125(4), 237-242 (1996).

8. Toda, M., "Wave propagation in anharmonic lattices," Journal of the Physical Society of Japan 23(3), 501-506 (1967).

9. Novoa, D., Malomed, B. A., Michinel, H., and Perez-Garcia, V. M., "Supersolitons: Solitonic excitations in atomic soliton chains," Phys. Rev. Lett. 101, 144101 (Sep 2008).

10. Taha, T. and Xu, X., "Parallel split-step fourier methods for the coupled nonlinear schrodinger type equations," The Journal of Supercomputing 32(1), 5-23 (2005).

11. Nickolls, J., Buck, I., Garland, M., and Skadron, K., "Scalable parallel programming with cuda," Queue 6, 40-53 (Mar. 2008). 